\title{
Coevolution signals capture the specific packing of secondary structures in protein architecture
}

\section{Dear Editor,}

Decoding protein structural characteristics and formation is essential to the understanding of its function. Despite the diversity and enormity of protein universe, the dissection of known protein structures has identified regular secondary structure elements (SSEs), namely, $\alpha$-helix and $\beta$-strand, that constitute the basic structural elements of protein universe (Levitt and Chothia, 1976). As a remarkable characteristics of the overall topology of a polypeptide, the specific spatial arrangement of these SSEs is a hallmark of the protein tertiary conformation (Murzin et al., 1995).

Unraveling the rules that govern the specific packing of the SSEs is critical to understanding the folding and formation of the tertiary structure of a protein and thus its function. Previously, numerous studies have attempted to identify the factors that determine the stability and specificity of SSE associations through analysis of super-secondary structure model systems like $\beta$-sheets and coiled-coils. Using designed $\beta$-hairpins or other $\beta$-sheet model systems, researchers have identified many factors that can significantly change the folding rate, stability or strand registry of $\beta$-sheets, such as charge interactions (Smith and Regan, 1995), beta propensity (Phillips et al., 2005) and $\beta$-sheet surface complementarity (Liang et al., 2008). The coiled-coil motif is another model system for understanding of molecular mechanisms that govern the super-secondary structure formation. Through NMR study of mutated GCN4p (Matousek et al., 2007) and design of stable and specific coiled-coils (Woolfson, 2005; Oakley and Kim, 1998), researchers have suggested the importance of electrostatic and polar interactions in determining the coiled-coil packing stability and specificity. In addition, Kennan and Ryan showed that changing the length of residue side chains could dramatically influence the stability of coiled-coils (Ryan and Kennan, 2007).

Despite the extensive attention on the factors that determine the specific packing of SSEs in certain forms, most of the above discoveries were made in simple soluble model systems but not natural proteins. Thus so far a global view of the effect of these factors on the protein topology still remains unclear. In this study, we would like to provide an evolutionary perspective into the roles of physiochemical properties in spatial arrangement of SSEs in protein universe. Based on a large ortholog group dataset, we systematically analyzed the coevolution signals of a variety of physiochemical properties for association of SSEs in protein evolution.

To analyze the extent of coevolution of two SSEs with regard to different physiochemical properties during protein evolution, we designed an analysis framework, which is illustrated in Fig. S1. In our analyses, the SSEs used threestate representations, namely helix, strand and loop. For physiochemical properties, we considered charge, volume, polarity, amphiphilicity, hydrophilicity and residue conformational preference namely beta propensity. For each target protein with known structure in the ortholog database OMA (the Orthologous MAtrix project) (Schneider et al., 2007), we made sequence alignment of the target protein to its homologous proteins. Then, the SSEs of homolog sequences denoted as homolog SSEs were assigned based on the aligned regions to the SSEs in the target protein. For each target SSE (i.e. SSE A in Fig. S1), the changes in a physicochemical property during evolution can be represented as a distance vector that consists of the differed values of the physiochemical property between the homolog SSEs and target SSE (vector A in Fig. S1). The differed value of a physiochemical property between a homolog SSE and its target SSE is the averaged difference of physiochemical properties over the aligned amino-acid residues (see Methods in Supplementary for details). Finally, the Spearman's rank correlation coefficient of the distance vectors of two target SSEs (i.e., SSE A and SSE B) was calculated as the coevolution score to indicate the extent of coevolution of the two target SSEs with regard to a physiochemical property. The design of the analysis framework presents a quantitative measure of the coevolution of SSEs in protein structures with regard to these general physiochemical properties, allowing us to understand the roles of these properties in directing the packing of SSEs during the protein folding.

To investigate how these physiochemical properties coevolve between two SSEs, we performed a large-scale analysis on the 29014 pairs of SSEs in contact derived from 


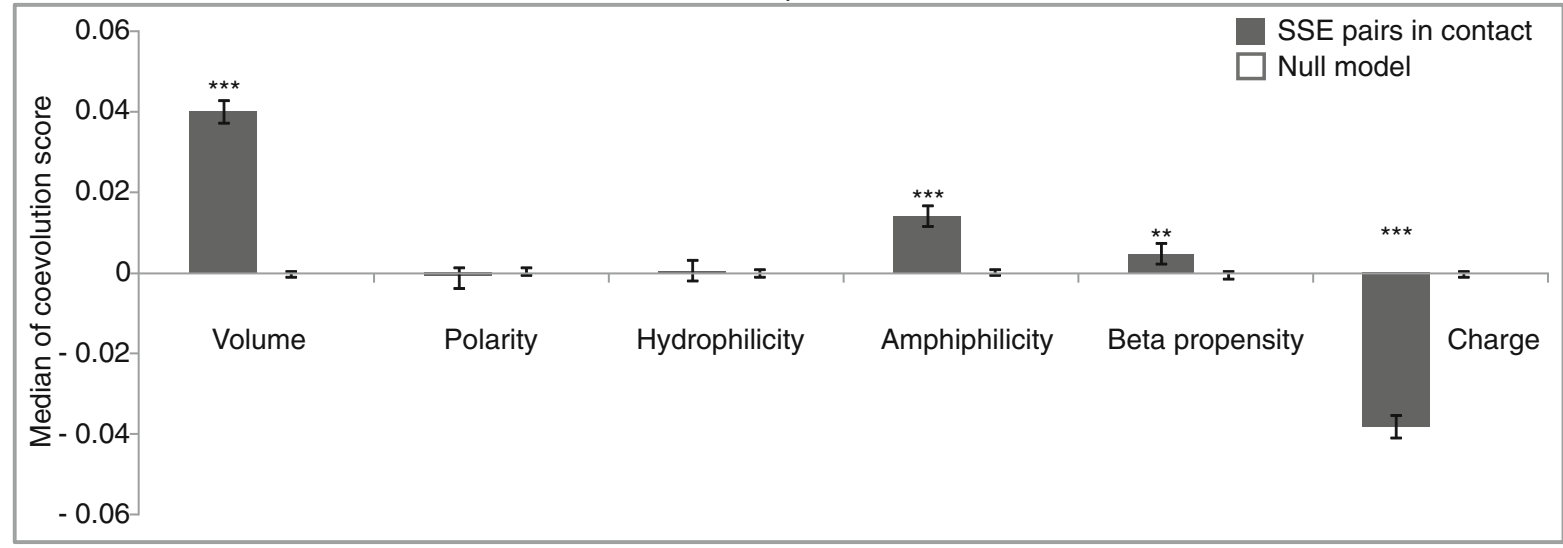

B

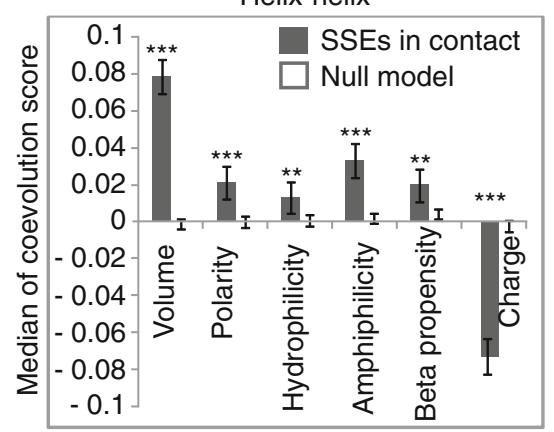

E

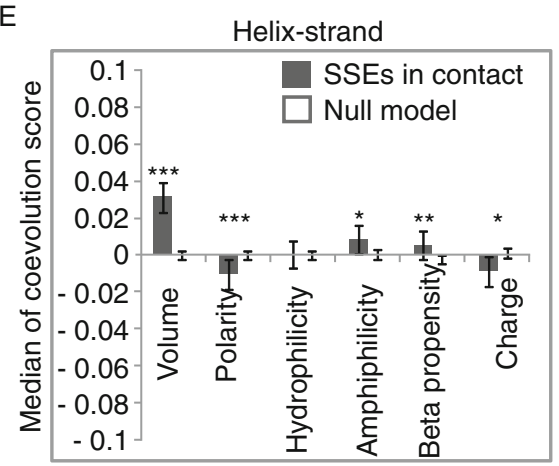

C

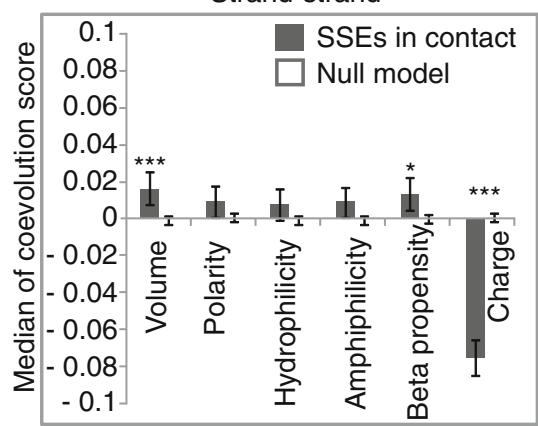

$\mathrm{F}$

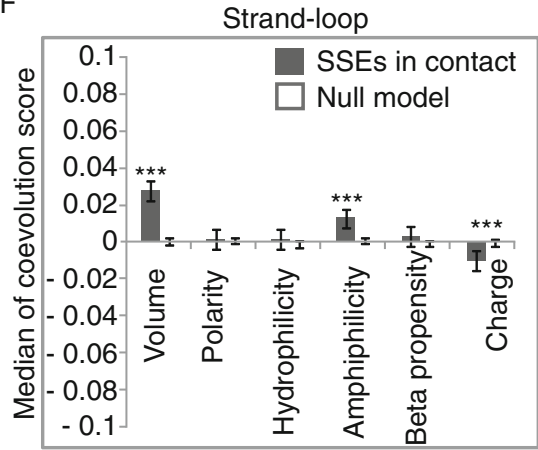

D Loop-loop

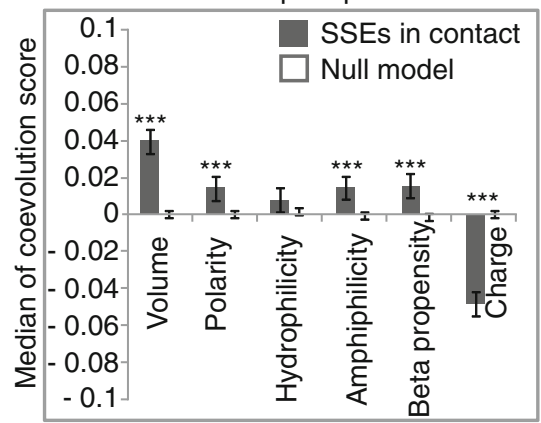

G

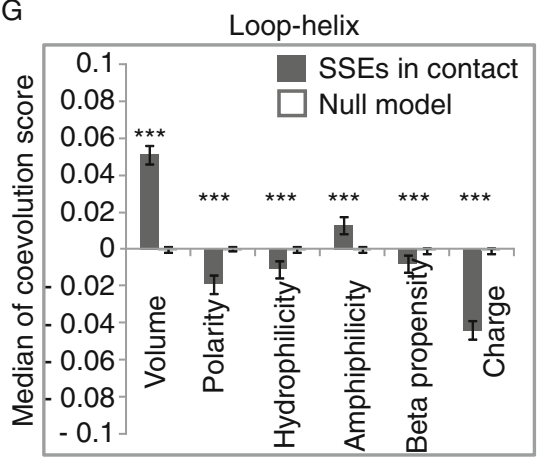

Figure 1. The coevolution signals of various physicochemical properties for SSEs in contact. (A) Comparison of coevolution signals of various properties for all types of SSE pairs in contact and random SSE associations (null model) (see Methods in Supplementary). (B-G) Comparison of coevolution signals of various properties between the SSE pairs in contact and the random SSE associations with regard to secondary structure types, for helix-helix packing (B), strand-strand packing (C), loop-loop packing $(D)$, helix-strand packing $(E)$, strand-loop packing $(F)$ and loop-helix packing $(G)$, respectively. For each property, the median values and the empirical $95 \%$ confidence intervals of coevolution scores (indicated by error bars) were plotted. ${ }^{*},{ }^{* *}$ and ${ }^{* * *}$ indicate level of significance according to Mann Whitney $U$-test: $P<0.05, P<0.01$ and $P<0.001$, respectively.

the 562 non-redundant ortholog groups collected from OMA database proteins (see Methods in Supplementary for details). For each physiochemical property, the coevolution scores of SSEs in contact were compared to the correlations of shuffled distance vectors (null model) (See Methods in Supplementary for details). Fig. $1 \mathrm{~A}$ shows the median score of SSEs in contact and random SSE associations for all SSE types regarding different physiochemical properties. Among the six physiochemical properties, the coevolution signals of four properties (charge, volume, amphiphilicity and beta propensity) showed a significant difference between SSE pairs in contact and random SSE associations $(P<0.01$ according to Mann-Whitney U-test. See Table S1 for details). Notably, the coevolution signal of charge property of SSEs in 
contact shifts significantly to the negative score relative to the random associations $\left(P<2.2 \times 10^{-16}\right)$. This negative correlation indicates that the coevolution of SSE charge property follows a complementary manner, underscoring the importance of maintaining the interactions between negative and positive charges in directing secondary structure packing (Acharya et al., 2006). While for volume, amphiphilicity and beta propensity, the coevolution scores of SSEs in contact are positive and significantly higher than those of random associations (volume: $P<2.2 \times 10^{-16}$, amphiphilicity: $P<2.2 \times 10^{-16}$ and beta propensity: $P<0.01$ ), suggesting the positively correlated changes of these properties for specific packing of SSEs during protein structure evolution.

Next, we sought to examine the contribution of different physiochemical properties to the specific packing of different types of secondary structures. It was found that for all types of SSE packing, the charge and volume properties presented significant coevolution signals, suggesting that charge and volume are two key players in the formation of protein topology (Fig. 1B-G). Interestingly, it was shown that the coevolution signals of these physicochemical properties differed significantly in the packing of different secondary structure types. For example, the helix-helix packing (Fig. 1B) and loop-helix packing (Fig. 1G) had significant coevolution signals in all of the six physicochemical properties surveyed, though the strength of these coevolution signals differed. In contrast, for strand-strand packing (Fig. 1C) and strand-loop packing (Fig. 1F), except for charge and volume, there has been observed only one property with significant coevolution signal, namely beta propensity for strand-strand packing and amphiphilicity for strand-loop packing. Moreover, though the underlying properties governing loop-loop packing (Fig. 1D) and helix-strand packing (Fig. 1E) are the same, the strength of the coevolution signals for these underlying properties differed. In a word, these analyses demonstrated that different physiochemical properties contribute differently to the packing of different secondary structure types.

Since the specific spatial arrangement of SSEs is a hallmark of the topology of a protein, accurate prediction of their specific packing will greatly improve protein structure modeling. Here we would like to demonstrate whether the robust coevolution signals of SSEs in contact can improve the prediction of their specific packing. As we know, the specific pairing of $\beta$-strands is one of the most important aspects of $\beta$-sheet proteins. However, due to the weak coevolution signal of residues within $\beta$-sheet, the coevolution information has not been considered directly in predicting $\beta$-strand pairing preference (Steward and Thornton, 2002; Cheng and Baldi, 2005; Lippi and Frasconi, 2009). Given the strong coevolution signals between $\beta$-strands in contact observed in our analysis, we tested whether the consideration of such coevolution information could improve $\beta$-strand pairing
Table 1. Improvement of strand pairing prediction by integrating the coevolution signals of $\beta$-strands

\begin{tabular}{lll}
\hline & Betapro & Betapro + coevolution \\
\hline Precision & $57.8 \%$ & $59.6 \%$ \\
Recall & $67.5 \%$ & $70.1 \%$ \\
F1 & $62.3 \%$ & $64.5 \%$ \\
\hline
\end{tabular}

10 -fold cross validation was performed on the dataset of 286 proteins extracted from Cheng and Baldi dataset with more than 200 homologs. F1 is the harmonic mean of precision and recall.

prediction. To simplify our test, we integrated the coevolution scores of the six physicochemical properties computed above into a well-known $\beta$-strand pairing predictor, BetaPro developed by Cheng et al. (see Methods in Supplementary for details) (Cheng and Baldi, 2005). In the test, we only considered the proteins with over 200 homolog sequences. Table 1 shows when the coevolution information is considered, the precision: $\mathrm{P}=\mathrm{TP} /(\mathrm{TP}+\mathrm{FP})$ and recall (also known as sensitivity): $\mathrm{R}=\mathrm{TP} /(\mathrm{TP}+\mathrm{FN})$ are improved by $1.8 \%$ and $2.6 \%$, respectively. The harmonic mean of precision and recall: $F 1=2 P R /(P+R)$ is improved by $2.2 \%$. Besides, as shown in Fig. S3, the AUC (area under the curve) of our model is 0.882 , presenting a significant improvement over the original BetaPro predictor of $0.864\left(P\right.$-value $=1.71 \times 10^{-4}$ by Delong's test (Delong et al. 1988)). Clearly, the incorporation of coevolution signal of $\beta$-strands can improve the prediction of their specific pairing in the formation of $\beta$ sheets, suggesting that the robust coevolutionary signals identified in our study can help us understand the molecular mechanisms underlying the formation of protein structure and thus its function.

In summary, we carried out a systematic analysis of coevolution of six general physiochemical properties between SSEs in contact within known protein structures, and revealed robust coevolution signals of different properties for the packing of different secondary structure types. In addition, the integration of coevolution information into protein structure prediction was found to slightly improve $\beta$-strand pairing prediction. Therefore, our work could not only shed lights into the molecular mechanisms governing specific packing of SSEs in protein structure formation, but also could facilitate protein structure prediction.

\section{FOOTNOTES}

We thank the members of Jiang lab for discussions. This study was supported by the National Basic Research Program (973 Program) (No. 2014CB910500), the National Natural Science Foundation of China (Grant No. 31371338) and National Science and Technology Major Project of the Ministry of Science and Technology of China (Grant No. 2013ZX10004611-002). 
Lizong Deng, Xiaoxi Dong, Aiping Wu, Tingrui Song and Taijiao Jiang declare that they have no conflict of interest. This article does not contain any studies with human or animal subjects performed by the any of the authors.

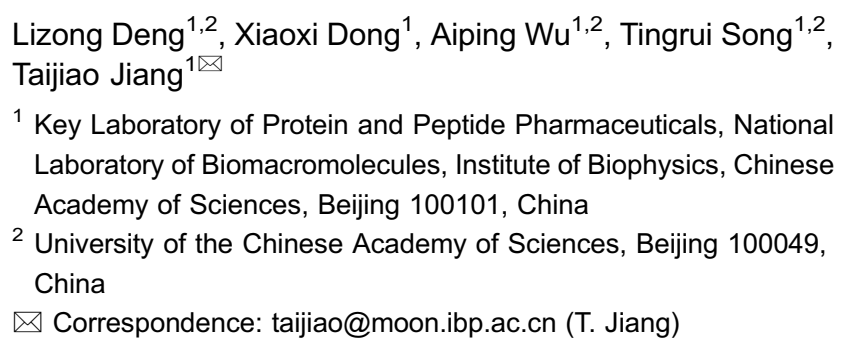

\section{OPEN ACCESS}

This article is distributed under the terms of the Creative Commons Attribution License which permits any use, distribution, and reproduction in any medium, provided the original author(s) and the source are credited.

\section{REFERENCES}

Acharya A, Rishi V, Vinson C (2006) Stability of 100 homo and heterotypic coiled-coil a-a' pairs for ten amino acids (A, L, I, V, N, K, S, T, E, and R). Biochemistry 45:11324-11332

Cheng J, Baldi P (2005) Three-stage prediction of protein betasheets by neural networks, alignments and graph algorithms. Bioinformatics 21(Suppl 1):i75-i84

DeLong ER, DeLong DM, Clarke-Pearson DL (1988) Comparing the areas under two or more correlated receiver operating characteristic curves: a nonparametric approach. Biometrics 44:837-845

Levitt M, Chothia C (1976) Structural patterns in globular proteins. Nature 261:552-558
Liang Y, Pingali SV, Jogalekar AS, Snyder JP, Thiyagarajan P, Lynn DG (2008) Cross-strand pairing and amyloid assembly. Biochemistry 47:10018-10026

Lippi M, Frasconi P (2009) Prediction of protein beta-residue contacts by Markov logic networks with grounding-specific weights. Bioinformatics 25:2326-2333

Matousek WM, Ciani B, Fitch CA, Garcia-Moreno B, Kammerer RA, Alexandrescu AT (2007) Electrostatic contributions to the stability of the GCN4 leucine zipper structure. J Mol Biol 374:206-219

Murzin AG, Brenner SE, Hubbard T, Chothia C (1995) SCOP: a structural classification of proteins database for the investigation of sequences and structures. J Mol Biol 247:536-540

Oakley MG, Kim PS (1998) A buried polar interaction can direct the relative orientation of helices in a coiled coil. Biochemistry 37:12603-12610

Phillips ST, Piersanti G, Bartlett PA (2005) Quantifying amino acid conformational preferences and side-chain-side-chain interactions in beta-hairpins. Proc Natl Acad Sci USA 102:13737-13742

Ryan SJ, Kennan AJ (2007) Variable stability heterodimeric coiledcoils from manipulation of electrostatic interface residue chain length. J Am Chem Soc 129:10255-10260

Schneider A, Dessimoz C, Gonnet GH (2007) OMA Browserexploring orthologous relations across 352 complete genomes. Bioinformatics 23:2180-2182

Smith CK, Regan L (1995) Guidelines for protein design-the energetics of beta-sheet side-chain interactions. Science 270:980-982

Steward RE, Thornton JM (2002) Prediction of strand pairing in antiparallel and parallel beta-sheets using information theory. Proteins-Struct Funct Bioinf 48:178-191

Woolfson DN (2005) The design of coiled-coil structures and assemblies. In: Parry DAD, Squire JM (eds) Fibrous proteins: coiled-coils, collagen and elastomers. Elsevier Academic Press Inc., San Diego, pp 79-112
Electronic supplementary material The online version of this article (doi:10.1007/s13238-014-0051-2) contains supplementary material, which is available to authorized users.

Lizong Deng and Xiaoxi Dong are contributed equally to this work. 